University of Nebraska - Lincoln

DigitalCommons@University of Nebraska - Lincoln

USDA National Wildlife Research Center - Staff Publications
U.S. Department of Agriculture: Animal and Plant Health Inspection Service

February 2004

\title{
Avoidance of plant secondary compounds by European starlings: citronellyls
}

Arla G. Hile

USDA/APHIS/WS, National Wildlife Research Center, c/o Monell Chemical Senses Center

Follow this and additional works at: https://digitalcommons.unl.edu/icwdm_usdanwrc

Part of the Environmental Sciences Commons

Hile, Arla G., "Avoidance of plant secondary compounds by European starlings: citronellyls" (2004). USDA National Wildlife Research Center - Staff Publications. 341.

https://digitalcommons.unl.edu/icwdm_usdanwrc/341

This Article is brought to you for free and open access by the U.S. Department of Agriculture: Animal and Plant Health Inspection Service at DigitalCommons@University of Nebraska - Lincoln. It has been accepted for inclusion in USDA National Wildlife Research Center - Staff Publications by an authorized administrator of DigitalCommons@University of Nebraska - Lincoln. 


\title{
Avoidance of plant secondary compounds by European starlings: citronellyls
}

\author{
Arla G. Hile* \\ USDA/APHIS/WS, National Wildlife Research Center, clo Monell Chemical Senses Center, 3500 Market St., Philadelphia, PA 19104, USA
}

Received 17 October 2003; received in revised form 18 February 2004; accepted 20 February 2004

\begin{abstract}
There is an ongoing need for nonlethal and environmentally safe vertebrate repellents for a variety of uses. One promising source of candidate repellents is plant secondary compounds, many of which have evolved to defend against invertebrate herbivory. In this study I tested six citronellyl compounds that are used in the human flavor and fragrance industry. Testing was conducted with an invasive and exotic avian pest species in North America, the European starling (Sturnus vulgaris). All six compounds tested were highly repellent to the birds and repellency was similar across treatment days. A dose-response experiment revealed that two of the six compounds, citronellyl acetate and citronellyl butyrate, tended to be repellent at lower concentrations (chow treated with 0.1:1 mixture of the citronellyl in ethanol) than the others (1:1 mixture). One compound, citronellyl valerate was also tested for within-day secondary repellency but no evidence was seen for this. It can be concluded that some plant derivatives are suitable for use as avian repellents and many other plant secondary compounds are promising resources for new and safe vertebrate repellents.
\end{abstract}

(C) 2004 Published by Elsevier Ltd.

Keywords: Aversion learning; Bird repellents; Citronellyl; Monoterpene; Sturnus vulgaris

\section{Introduction}

There is an ongoing and increasing need for the development of new pest control methods to protect agricultural and natural resources. The need is greatest for nonlethal control methods because of societal demands, the requirements of conservationally-sound management, and ethical concerns. Many chemicals previously available for use are no longer available, primarily because of ecological or human safety reasons.

Repellents can operate via two mechanisms: primary or secondary repellency. Primary repellents are those that are immediately aversive to the animal, causing a rapid response. In this case, a chemically mediated cue from the repellent (flavor or irritation) is the unconditioned stimulus, resulting in an unconditioned withdrawal response. Primary repellents used alone do not promote long-term avoidance; instead animals selfregulate and thereby limit their exposure to the repellent (Domjan, 1998; Sayre and Clark, 2001). Furthermore,

\footnotetext{
*Corresponding author. Tel.: + 1-215-898-5753; fax: + 1-215-8982084.

E-mail address: arlahile@monell.org (A.G. Hile).
}

many primary repellents degrade rapidly under field conditions (Aronov and Clark, 1996). As currently used, primary repellents are ineffective without frequent reapplication.

Despite their limitations, primary repellents remain an important means to control wildlife. They tend to be environmentally safe (Mason and Clark, 1992), do not require animals to learn (Clark, 1998), and they are at least modestly effective (Sayre and Clark, 2001). Other than exploring new candidate repellents, there might be ways to improve the effectiveness of existing repellents. For example, one unexplored means to increase the effectiveness of primary repellents would be to increase their salience to target animals (Sayre and Clark, 2001), particularly by pairing them with methods that increase the stress hormone levels of the target species (Hile, in press). Finally, some primary repellents might also work secondarily by inducing malaise (Sayre and Clark, 2001).

Secondary repellents function as a result of their systemic effects; typically these effects appear to be gastrointestinal distress or general malaise. The illness caused by secondary repellents is the unconditioned stimulus; this stimulus can then be paired with 
conditioned stimuli such as some feature of the repellent or additional cues (Revusky, 1977). Secondary repellents are more effective than primary repellents because they promote long-term avoidance responses that are proportional to the severity of the malaise caused by the compound. However, many secondary repellents or their degradation products can have undesirable side effects on the target species (Dolbeer et al., 1994), consequently the use of many secondary repellents such as methiocarb is falling out of favor. New secondary repellents need to be found that are safer for the environment and the target organisms, a task that thus far has proved difficult.

Testing candidate repellents has relied on behavioral assays, which are tedious, inefficient, and time-consuming. Efforts have been made to streamline this process, for example, by analyzing the relationship between chemical structure and repellency (Clark and Shah, 1994), by employing cell cultures to rapidly screen chemicals (Bryant, 1997), or by considering chemical ecology for existing natural repellents. One potential source for candidate repellents is from plant secondary compounds. Some plant secondary compounds are already approved as human food flavorants, which may ease licensing them for use under increasingly stringent EPA environmental standards (Fagerstone and Schafer, 1998). Data collected using a structureactivity approach have lead to studies focusing on sixmember, ringed aromatic compounds (Watkins et al., 1999). Here I describe tests of six citronellyl compounds on a vertebrate species introduced to North America for which there exists a large database on chemical sensitivity, the European starling (Sturnus vulgaris).

\section{Methods}

\subsection{Subjects}

European starlings of unknown age were trapped during October 2002 using baited funnel traps in Doylestown, PA and transported to the laboratory. During the first 5 weeks in the laboratory the birds were screened for diseases and their weights were monitored twice weekly. Subjects were maintained on a $12 \mathrm{~h}: 12 \mathrm{~h}$ L:D photoperiod under broad-spectrum fluorescent lighting, fed an ad libitum diet of a commercial passerine chow, tap water, and received fruit and mealworms (Tenebrio larvae) once a week. After the first 5 weeks of captivity, the appearance of all animals was monitored daily and their weights checked weekly. Subjects were housed in a group cage until 2 weeks prior to the onset of the experiment.

Two weeks prior to the onset of the study, 24 birds were placed into individual cages measuring $33 \times 33 \times 61 \mathrm{~cm}^{3}$ and provided with cat toys for envir- onmental enrichment that were changed weekly. At the same time, birds were switched to a diet of passerine chow that had been soaked for $5 \mathrm{~min}$ in $95 \%$ ethanol, then strained and allowed to dry overnight. This was done so that the birds would become accustomed to the solvent that would be used for the citronellyls. Food was provided in partially-covered stainless steel cups with partial lids to minimize spillage. Any bird that lost more than $8 \%$ of its body weight during the first week following the change in housing and diet was removed from the study and replaced by a different bird.

\subsection{Group assignment}

The baseline consumption levels of experimental subjects were measured in pre-testing as follows: at $1600 \mathrm{~h}$ the food bowls were removed from the birds' cages. The following morning, at $0900 \mathrm{~h}(2 \mathrm{~h}$ after lights on), each bird was presented with $25 \mathrm{~g}$ of the ethanoltreated chow. At $1200 \mathrm{~h}$, the remaining food was removed and weighed. This was repeated the next day. Water was always available. Birds were then divided into low, moderate, and high-consumption categories (8 in each category). Two birds from each consumption category were assigned to each of four treatment groups ( $n=6$ per group). One-way ANOVA confirmed that there was no difference in baseline food consumption among the four treatment groups $(P>0.20$ for the 2 days combined).

\subsection{Test compounds}

Six compounds were tested, all were citronellyls: citronellyl butyrate, citronellyl isobutyrate, citronellyl valerate, citronellyl acetate, citronellyl formate and citronellyl propionate (Fig. 1). These were obtained from Sigma-Aldrich (Milwaukee, WI) and sold in solution that ranged from $85+\%$ to $90+\%$ purity by weight; the remainder of each solution consisted of impurities that were primarily isomers of the main component. Each compound was determined to be miscible in $95 \%$ ethanol, in which it was dissolved to make a solution for treating food.

\subsection{Experiment 1}

A 1:1 (by weight) ratio of each compound (based on percentage by weight of the primary component) to $95 \%$ ethanol was prepared and this mixture was used to treat the standard chow using the same method as for the ethanol-treated chow. At $0900 \mathrm{~h}$ following overnight food deprivation birds were presented with $50 \mathrm{~g}$ of the treated chow in a one-choice feeding test. Three groups of six birds were given food treated with a test compound while the fourth group served as a control (these birds received ethanol treated food). Water was 


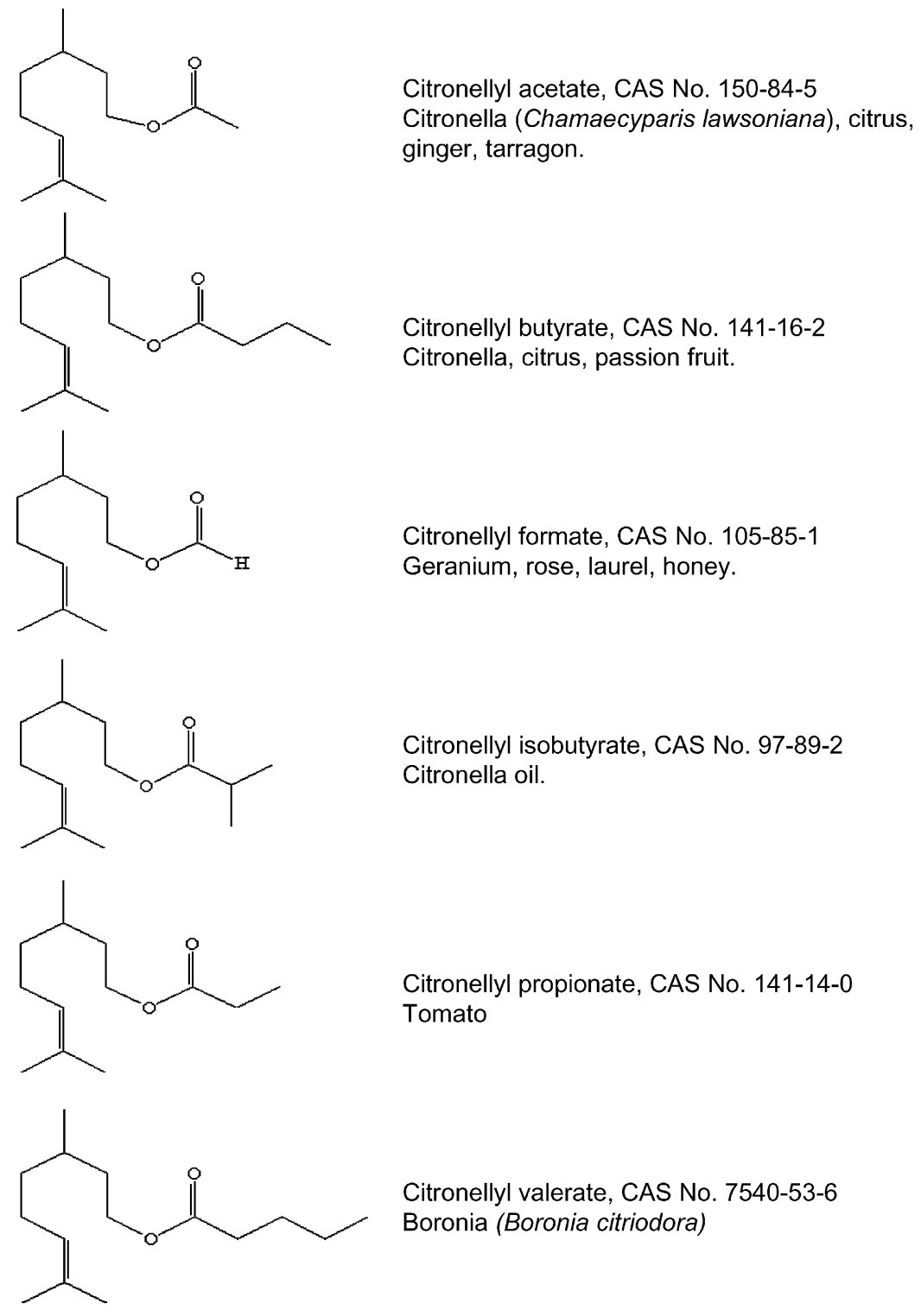

Fig. 1. Chemical structures, CAS numbers, and some known sources for the compounds tested in the current study.

always available during testing. After $3 \mathrm{~h}(1200 \mathrm{~h})$, the treated chow was removed and replaced by the standard, ad libitum diet. Testing was repeated 7 and 14 days later. One week after the day 14 trial, the experiment was repeated with the three remaining compounds and using the same groups of subjects.

\subsection{Experiment 2}

Four of the test compounds decreased consumption by approximately $90 \%$ at the $1: 1$ concentration in ethanol; therefore, a second experiment was conducted with these four compounds to measure the dosedependent response. Each compound was mixed in $95 \%$ ethanol in a series of dilutions by weight of compound:ethanol as follows: 0.1:1, 0.01:1, 0.001:1 and $0.0001: 1$. Each concentration of each compound in alcohol was used to treat the standard chow as described in the methods of experiment 1. For each test compound, each of the four concentrations of treated chows was presented to one group of six birds $(n=6$ per concentration). Birds were allowed at least 1 day off between testing of each concentration series.

\subsection{Experiment 3}

One of the six compounds, citronellyl valerate, had greater repellency on the day 7 and 14 trials than on the initial trial, suggesting that it may have secondary repellency effects. Therefore, an experiment was conducted to monitor hourly consumption over the course of $6 \mathrm{~h}$. Citronellyl valerate:ethanol treated chow (1:1 by weight) was tested as described previously. Following overnight food deprivation, six birds were presented 
with bowls containing $50 \mathrm{~g}$ of the treated chow. Every hour for the following $6 \mathrm{~h}$ the amount of food eaten was measured by quickly weighing the bowls. The bowls were then returned to the cages until all $6 \mathrm{~h}$ of trial was complete.

\subsection{Analysis}

Repeated measures ANOVA was used to analyze the data from experiments 1 and 3 , while a simple one-way ANOVA was sufficient for the experiment two dataset. Fisher's LSD test was used for the post-hoc analyses. All analyses were conducted using Statistica 6 software.

\section{Results}

\subsection{Experiment 1}

Three compounds were tested at a time, and for these two periods of experimentation there was no significant difference in the consumption levels of the control birds $(P>0.20)$. Consequently the data for all six compounds, along with the two groups of control animals, were pooled and analyzed as one experiment. All of the six compounds caused a significant reduction in food consumption compared to controls over the 3 test days (effect of treatment group across all days, $F_{6,41}=99.14$, $P<0.0001)$. There was also a significant interaction across days and the treatment group $\left(F_{12,82}=3.14\right.$, $P=0.001)$ due to the differential response to citronellyl valerate alone across treatment days. Overall, there was an $84.3 \%$ decrease in food consumed across all compounds and test days. Post hoc analysis revealed a difference between the two most repellent compounds and the remaining four. Specifically, citronellyl isobutyrate and citronellyl valerate caused roughly a $71 \%$ reduction in consumption by the birds, while the remainder caused an approximately $91 \%$ reduction in consumption (Fig. 2).

There was no effect of treatment day; however, there was greater avoidance of chow treated with citronellyl valerate between day 0 and the two subsequent test days. On day 0 , birds ate $1.75 \pm 1.39 \mathrm{~g}$ of the citronellyl valerate-treated chow, while across the next two treatment days they ate only $0.575 \pm 0.531 \mathrm{~g}$ of the same chow. This is consistent with a learned avoidance response, and suggests that citronellyl valerate may be a secondary repellent.

\subsection{Experiment 2}

Further testing of the four strongest primary repellents revealed that all were less effective at lower concentrations (Fig. 3). There was a non-significant trend for two of the repellents, citronellyl butyrate and citronellyl acetate, to be somewhat effective at the 0.1:1 concentration (when compared to data from experiment

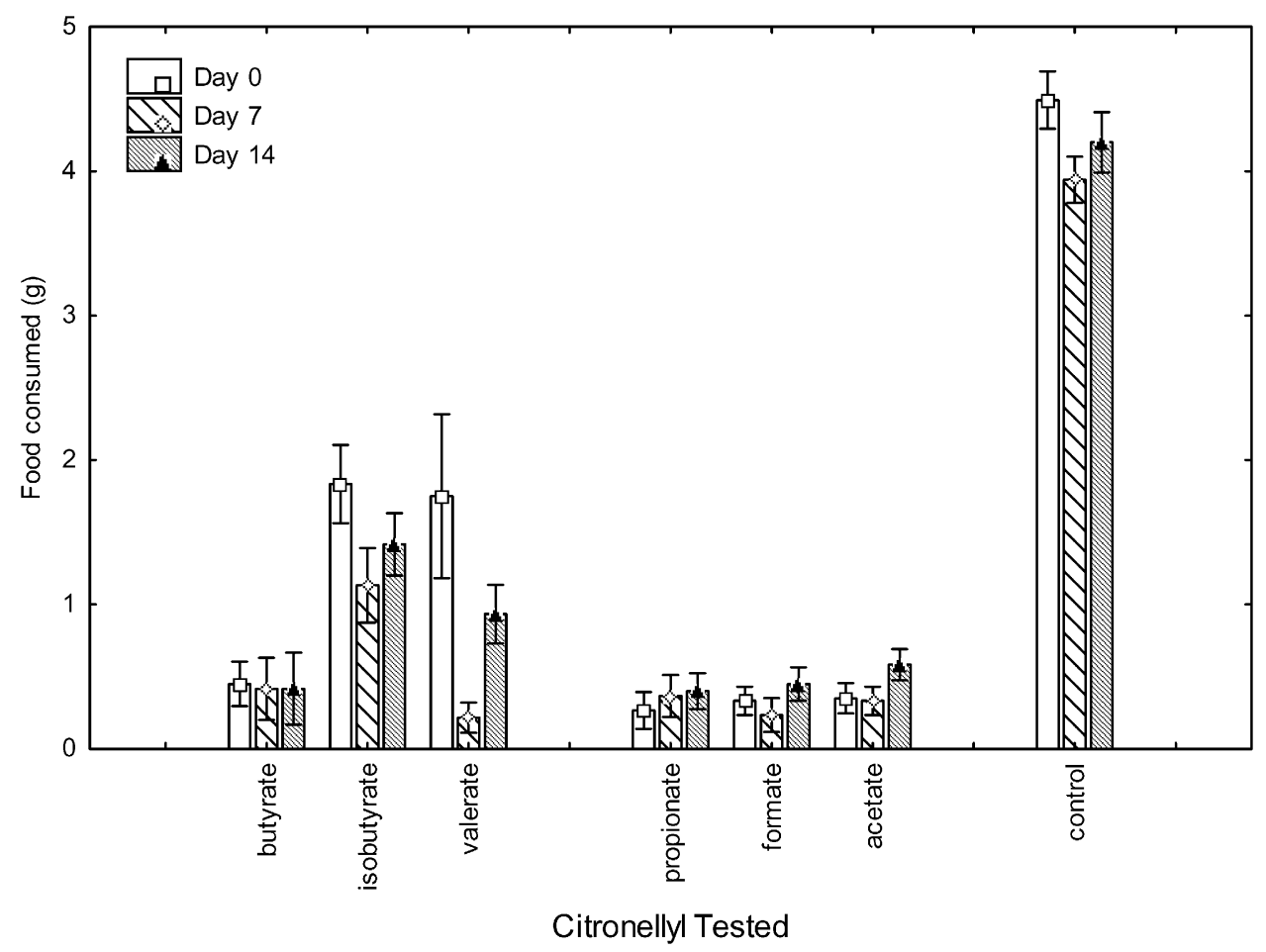

Fig. 2. Means and standard errors of grams of treated food consumed during a 3-h period across three testing days for the six tested compounds and control animals. Consumption was significantly lower when animals were presented with treated food as compared to controls, $F_{6}, 41=99.14$, $P<0.0001$. 


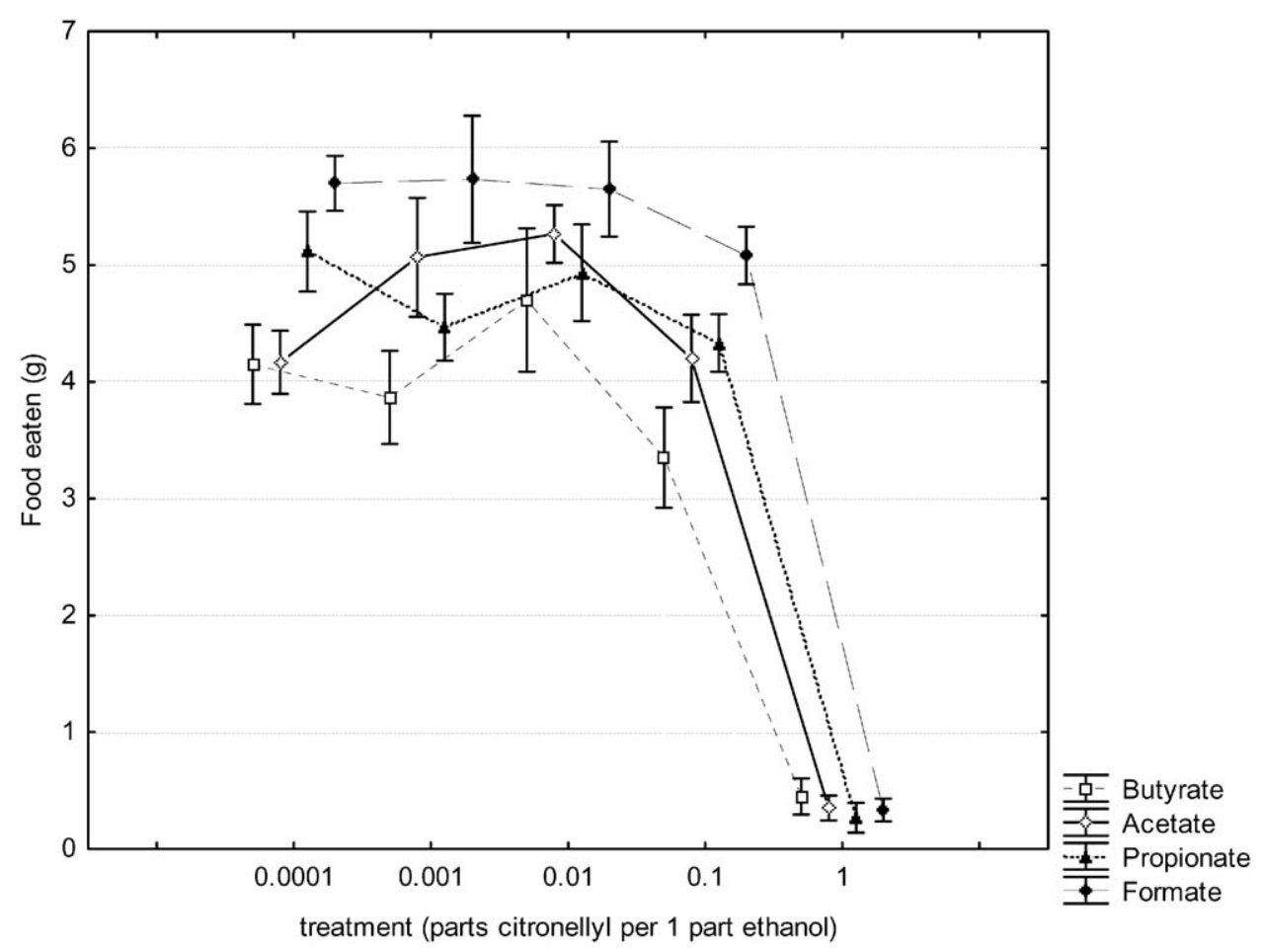

Fig. 3. Dose-response curves for the four most repellent compounds. Data from experiment 1 has been appended to the graph (1 part citronellyl in ethanol).

one controls, $P=0.064$ and 0.082 , respectively). Additional testing at concentrations between 0.1 and 1 part citronellyl per part alcohol and in more natural (field) conditions using these compounds is needed.

\subsection{Experiment 3}

There was no decrease in the hourly consumption rates of citronellyl valerate during the $6 \mathrm{~h}$ of the test (Fig. 4). While the possibility remains that there are secondary repellency effects of this compound, the time frame during which this occurs would be longer than the $6 \mathrm{~h}$ period used during this experiment.

\section{Discussion}

Of the six compounds tested, four were associated with a $91 \%$ decrease in food consumption, two caused a $71 \%$ decrease in food consumption, and one might have had some secondary repellency over multi-day time periods.

Specifically, in experiment 1 all of the compounds were significantly repellent at the relatively high concentrations tested. Because the number of available birds was limited, each subject was used to test two different candidate repellents, however the consistent results across all compounds indicates that there was not an effect of previous testing on the later tests. The

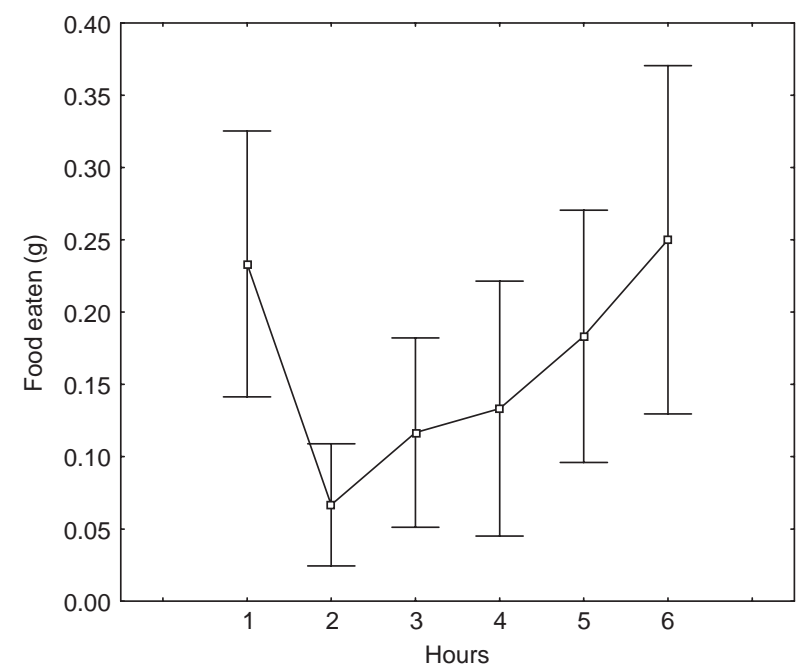

Fig. 4. Means and standard errors of food eaten per hour by birds presented with chow treated with a 1:1 mixture of citronellyl valerate and $95 \%$ ethanol by weight.

conclusion from experiment 1 is that all of the tested compounds are repellent at high concentrations.

Of the four strongest repellents, however, two might be effective at a lower concentrations. These compounds, citronellyl butyrate and citronellyl acetate, show promise as avian chemical repellents and should be evaluated further with different species and under different conditions. Testing should be conducted under field conditions and over longer periods of time. While 
the results from experiment 3 did not demonstrate that citronellyl valerate is a secondary repellent, there may be effects on feeding behavior across longer time intervals, as suggested from the results of experiment 1 .

The mechanism by which these repellents function is unknown, however they are structurally similar to compounds that are trigeminal irritants (L. Clark pers. comm.). Trigeminal irritation would also be consistent with the observation that these compounds function as primary repellents. However, these compounds might also function as secondary repellents if they were somehow delivered into the gastrointestinal tract without stimulating the trigeminal receptors in the mouth (perhaps through encapsulating them). Secondary repellency has been demonstrated previously with a known primary repellent, methyl anthranilate delivered via intubation (Sayre and Clark, 2001).

A remarkable finding of this study is that all of the compounds were similarly repellent to starlings, despite the large differences in their chemical sidechains. Additional plant secondary compounds should be screened for their repellent effects on birds and other taxa. Clearly they can function on a range of species beyond the invertebrate herbivores under whose selection they evolved. Because many of these are already approved for human consumption, they offer a promising alternative pest control method that is economical and safe for humans and the environment.

Finally, why these compounds are repellent to birds is somewhat counterintuitive. All are considered attractive enough to humans to be used in products intended for human use (Burdock, 2001). Many are derived from fruits that are clearly attractive not only to humans, but to other animals including birds. In both their natural and isolated state, however, they are combined at low concentrations with other compounds which may interact with them in a way that makes them attractive. This implies that citronellyls or any other plant secondary compound that has repellent properties will likely have to be applied in high concentrations and on particular substrates in order to be effective. Testing in additional species and in the field would be an appropriate next step for these compounds.

\section{Acknowledgements}

I thank Stanley Lewis for technical assistance in data collection and Larry Clark for general suggestions and insightful comments on the data. Robert Beason provided valuable assistance in drafting the manuscript.

\section{References}

Aronov, E.V., Clark, L., 1996. Degradation studies of the non-lethal bird repellent, methyl anthranilate. Pestic. Sci. 47, 355-362.

Bryant, B.P., 1997. Peripheral trigeminal neural processes involved in repellency. In: Mason, J.R. (Ed.), Repellents and Wildlife Management. Colorado State University Press, Fort Collins, CO, pp. 19-27.

Burdock, G.A., 2001. Fenaroli's Handbook of Flavor Ingredients, 4th Edition. CRC Press, London.

Clark, L., 1998. Physiological, ecological, evolutionary bases for the avoidance of chemical irritants by birds. Curr. Ornithol. 14, $1-37$.

Clark, L., Shah, P., 1994. Tests and refinements of a general structure-activity model for avian repellents. J. Chem. Ecol. 20, 321-339.

Dolbeer, R.A., Avery, M.L., Tobin, M.E., 1994. Assessment of field hazards to birds from methiocarb applications to fruit crops. Pestic. Sci. 40, 147-161.

Domjan, M., 1998. The Principles of Learning and Behavior, 4th Edition. Brooks/Cole, Pacific Grove, CA, USA.

Fagerstone, K.A., Schafer Jr, E.W., 1998. Status of APHIS vertebrate pesticides and drugs. Proc. Vert. Pest Conf. 18, 319-324.

Hile, A.G., 2003. Fear and loathing: the modulatory effect of stress on memory formation and its wildlife management implications. Proceedings of the Bird Strike Commitee USA/Canada 2003 Conference. Accessed 3/2004 at http://www.birdstrike canada.com/ Papers 2003/Arla\% 20 Hile. doc.

Mason, J.R., Clark, L., 1992. Nonlethal repellents: the development of cost-effective, practical solutions to agricultural and industrial problems. Proc. Vert. Pest Conf. 15, 115-129.

Revusky, S., 1977. Learning as a general process with an emphasis on data from feeding experiments. In: Milgram, N.W., Krames, L., Alloway, T.M. (Eds.), Food Aversion Learning. Plenum Press, New York, pp. 1-5.

Sayre, R.W., Clark, L., 2001. Effect of primary and secondary repellents on European starlings: an initial assessment. J. Wildl. Manage. 65, 461-469.

Watkins, R.W., Lumley, J.A., Gill, E.L., Bishop, J.D., Langton, S.D., MacNicoll, A.D., Price, N.R., Drew, M.G.B., 1999. Quantitative structure-activity relationships (QSAR) of cinnamic acid bird repellents. J. Chem. Ecol. 25, 2825-2845. 